TITLE:

\title{
PATTERN OF BLASTULA FORMATION IN SEA URCHIN DEVELOPMENT
}

$\operatorname{AUTHOR}(S)$ :

Maruyama, Yoshihiko K.; Satoh, Noriyuki

\section{CITATION:}

Maruyama, Yoshihiko K....[et al]. PATTERN OF BLASTULA FORMATION IN SEA URCHIN DEVELOPMENT. PUBLICATIONS OF THE SETO MARINE BIOLOGICAL LABORATORY 1977 , 24(1-3): 1-8

ISSUE DATE:

1977-11-30

URL:

http://hdl.handle.net/2433/175966

RIGHT: 


\title{
PATTERN OF BLASTULA FORMATION IN SEA URGHIN DEVELOPMENT ${ }^{1,2)}$
}

\author{
Yoshiniko K. MARUYAMA and NoRIYUki SATOH \\ Department of Zoology, Faculty of Science, Kyoto University, Kyoto 606, Japan
}

With Text-figures $1-20$

\section{Introduction}

The formation of the blastula is the earliest morphogenetic event in echinoderm development in which a group of cells become organized in a well-defined form (Wolpert and Gustafson, 1961). During blastula formation the cells of sea urchin embryos form a single layer enclosing the blastocoel, which increases in size, the whole making a hollow sphere. The blastocoel formation and the volume increase of the embryo can be thought of one kind of growth, which leads and/or ensures the following processes of development such as emigration of the primary mesenchyme cells and gastrulation. Several hypotheses have been put forward to account for this process, the most detailed being that of Dan (1952, 1960). He has suggested, based on the observation of an inflation of the hyaline layer immediately before cleavage in Mespilia globulus and by the experiment using the eggs developing in gum arabic, that the enlargement of blastocoel is largely due to the adherence of the cells to the hyaline layer and the accumulation of fluid, initially within this layer, due to increased colloid osmotic pressure, and later within the blastocoel. He has also pointed out that the enlargement of blastocoel occurs in stepwise fashion in connection with the each cleavage cycle until the 7 th cleavage in Mespilia globulus. Monné and Hårde (1951) have indicated that the blastocoel is filled with a mucoid jelly secreted by blastomeres and have also suggested an osmotic mechanism for inflating the blastocoel. On the other hand, Wolpert and Gustafson (1961) have observed in Psammechinus miliaris that at the time of cleavage there is a large increase in blastocoel volume, but at the following interphase the volume is reduced. They have pointed out that it is very difficult in terms of Dan's osmotic theory to account for the reduction in volume following cleavage and have postulated that the enlargement of blastocoel in connection with each cleavage is due to the reduction in thickness of the blastula wall at successive cleavages.

1) This paper is dedicated to the memory of the late Professor KEN-ITI SIRAKAMI (1913-1974) of the Kyoto University.

2) Contributions from the Seto Marine Biological Laboratory, No. 621.

Publ. Seto Mar. Biol. Lab., XXIV (1/3), 1-8, $1977 . \quad$ (Article 1) 
The above quoted studies were carried out on the species which yield the formation of a hollow sphere with single cell layer mainly at the cleavage stage. In some species of sea urchins such as Anthocidaris crassispina, however, the blastocoel is hardly noticeable even at the time of hatching and an increase in embryonic volume takes place after hatching. The aim of the present study is to make clear the temporal pattern of blastula formation in several species of sea urchins. Therefore, the main concern here is with the time of appearance of the intra-embryonic space and its magnitude rather than with the detailed mechanisms by which such changes may be brought about. The result indicates that differnet patterns of the blastula formation in seven species of sea urchins can be classified into three types, which are, as a result, in accordance with the taxa at the family level. The present result may be valid for further discussions on the mechanism of blastula formation as well as on the taxonomical correlation among the seven species.

\section{Materials and Methods}

Seven species of sea urchins (Mespilia globulus, Temnopleurus reevesi, Toxopneustes pileolus, Tripneustes gratilla, Pseudoboletia maculata, Anthocidaris crassispina, and Echinometra mathaei) were collected on the coast near the Seto Marine Biological Laboratory, during the presumed breeding season, from the middle of July through August in 1975 and 1976. Fertilized eggs were obtained by the usual method and were allowed to develop at $26.0-27.0^{\circ} \mathrm{C}$. Developing embryos were photomicrographed and time-lapse cinematographed from the time of fertilization until hatching. After hatching parts of blastulae were killed at appropriate intervals with $0.01 \%$ glutaraldehyde in sea water and the fixed specimens were photomicrographed. The difference in the embryonic volume between living and fixed materials was checked to be negligible. In all the observations the plane of focus was the maximum optical cross-section. Time-lapse cinematographs $(16 \mathrm{~mm})$ were made at $8 \mathrm{sec}$ intervals with a Nikon CFMA equipped with a Bolex H-16 camera and were analysed with the aid of a Nac Dynamic Frame Analyser. Accurate tracings of the embryos were made by projecting individual frames on paper. For an estimation of the embryonic volume, diameters of fertilization membrane, embryo and blastocoel were measured respectively in at least three directions on paper in the case of time-lapse cinematography and on printing paper in the case of photomicrography. Based on the assumption that the embryo is a sphere until the late blastula stage, respective volumes were calculated by the average diameters. Alternatively, these volumes were estimated on the basis of the cross-sectional areas measured by planimetry. In each species studied, the estimation of the volume at every time point was consisted of at least 10 individual embryos.

\section{Results}

As shown in Figures 1-19, patterns of the blastula formation were considerably 
different among the seven species of sea urchins studied. An extensive analysis, however, made it possible to classify three different types of blastula formation according to the temporal ordering: the pre-hatching type which includes Mespilia globulus and Temnopleurus reevesi; the post-hatching type to which Anthocidaris crassispina and Echinometra mathaei belong; and the continuous type, Toxopneustes pileolus, Tripneustes gratilla and Pseudoboletia maculata. The three types classified were, as a result, in accordance with the taxa at the family level. The pre-hatching type is for the
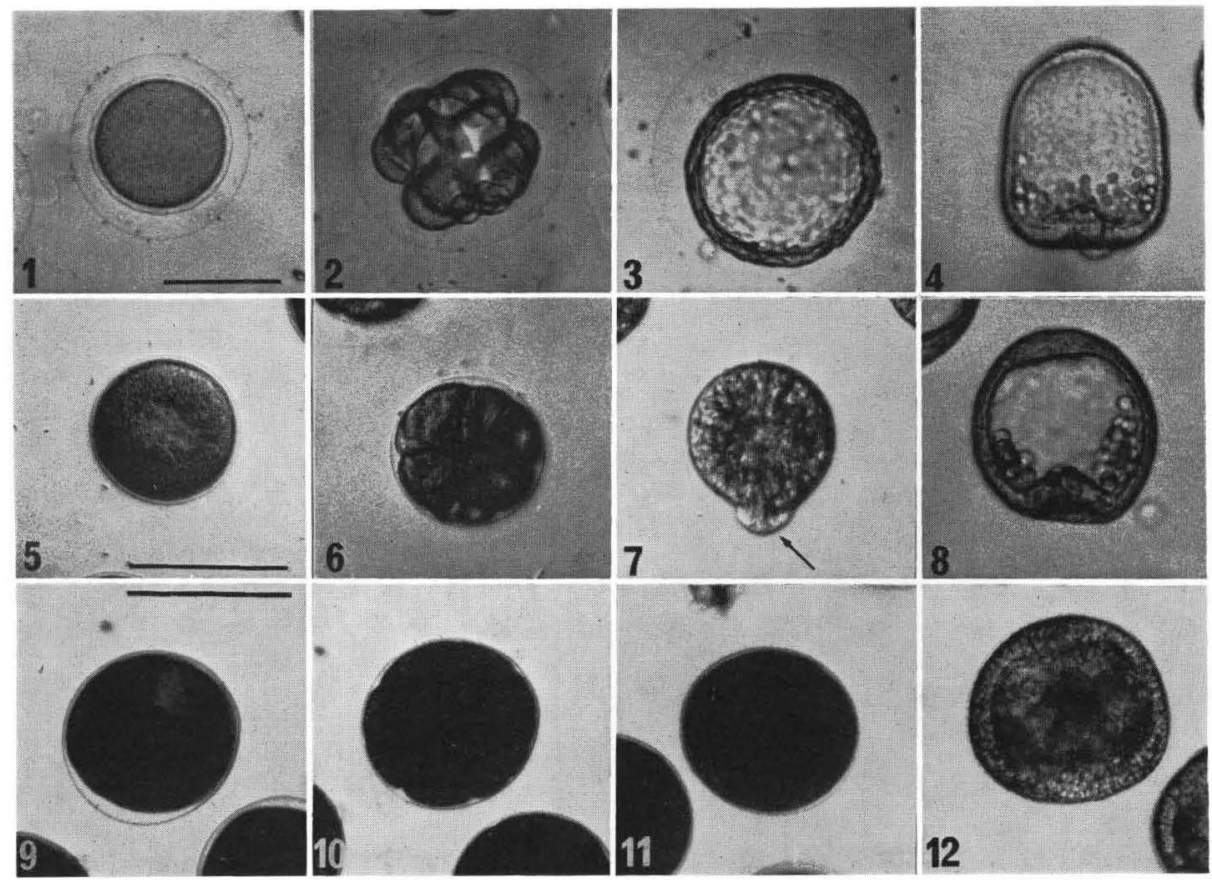

Fig. 1-12. Three different patterns of blastula formation (pre-hatching, continuous and posthatching type) of sea urchin embryos are shown in serial microphotographs. The upper row (1-4) indicates the blastula formation in Mespilia globulus (the pre-hatching type), the middle row (5-8) in Tripneustes gratilla (the continuous type) and the lower row (9-12) in Anthocidaris crassispina (the post-hatching type). The developmental stages are, from the left hand, the uncleaved stage $(1,5,9)$, the 16-cell stage $(2,6,10)$, the pre-hatching stage $(3,7,11)$, and the initial gastrulation stage $(4,8,12)$, respectively. The increase of embryonic volume is exclusively due to that of blastocoel. Hatching in Tripneustes begins by protrusion of a certain portion of embryonic wall through the fertilization membrane (7, arrow). Bar, $100 \mu \mathrm{m}$.

family Temnopleuridae, the post-hatching type for the family Echinometridae, and the continuous type for the family Toxopneustidae, respectively.

The Pre-hatching type: In Mespilia and Temnopleurus, the formation of blastocoel was completed during the cleavage stage. The blastocoel was detected at the first cleavage. A large and rapid increase both of embryo and blastocoel volume took place from the first cleavage up to the 7th cleavage cycle (Figs. 1-4, 13, 14). The enlargement of the blastocoel occurred during the interphase of each cleavage 

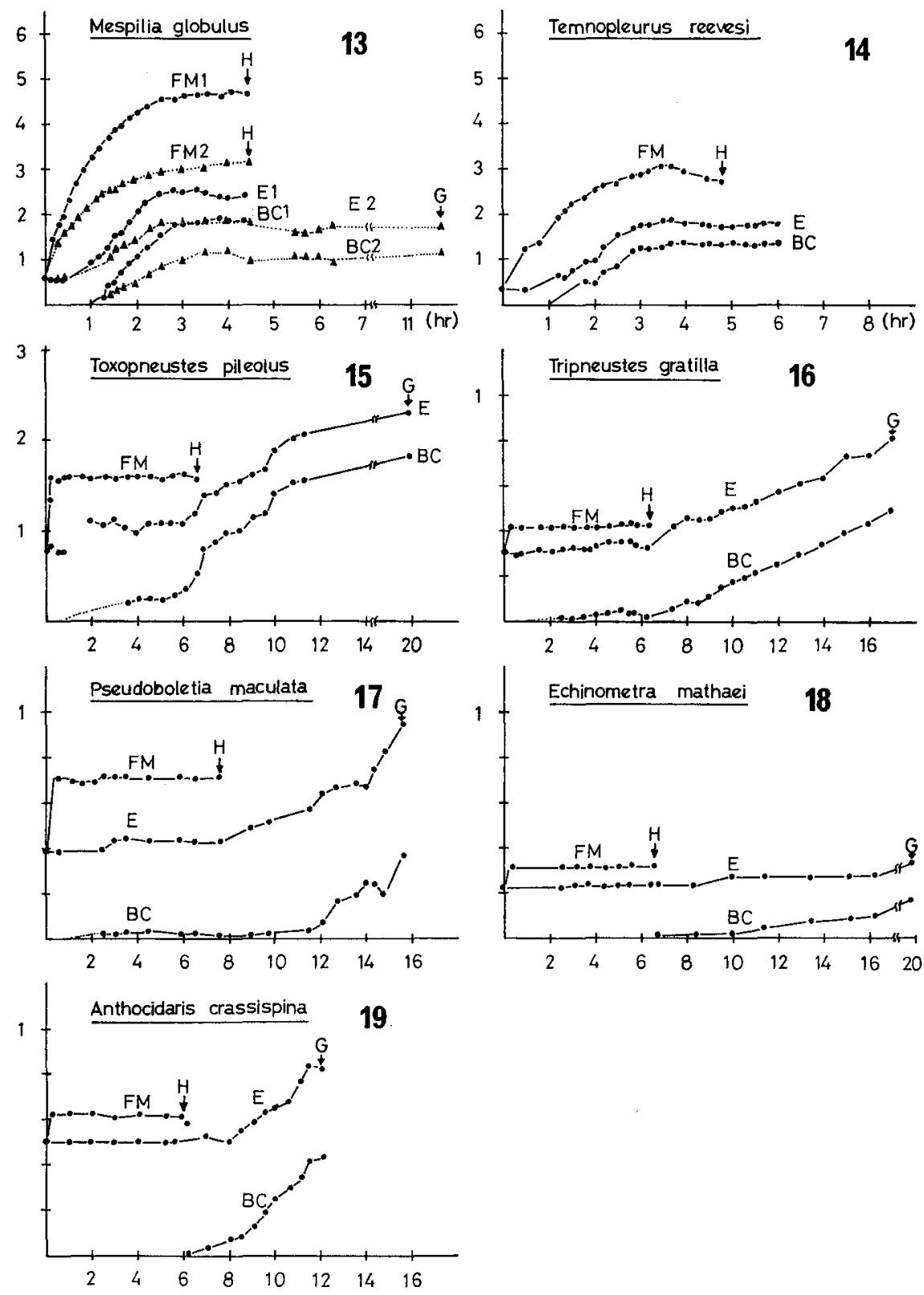

Figs. 13-19. Change in the volume enclosed by fertilization membrane (FM), of embryo (E) and of blastocoel (BC) during blastula formation in seven species of sea urchins. Abscissa; time after fertilization (hour). Ordinate; the volume $\left(\times 10^{6} \mu \mathrm{m}^{3}\right) . \mathrm{H}$; time of hatching. G; gastrulation. Each point in the figure is the average value of at least 10 embryos obtained from the same pair. In Mespilia (13) two samples at different times in a breeding season are indicated. 
cycle in step-wise fashion (Maruyama, 1977). Thereafter, the volumes did not increase until hatching (Figs. 1-4, 13, 14). After hatching further increase both of embryo and blastocoel volume occurred very slightly (Figs. 13, 14).

As the fertilization membrane continued to elevate from the time of fertilization to the first cleavage, the volume of perivitelline space increased until the first cleavage. Although the volume enclosed by the fertilization membrane yielded further increase up to the end of the 7 th cleavage cycle (Figs. 1-3, 13, 14), the volume of perivitelline space remained rather constant during this period, which seemed to indicate that the enlargement of the region enclosed by the fertilization membrane was exclusively due to the volume increase of embryo.

The POST-HATGHING TYPE: In contrast to the pre-hatching type, the fertilization membrane of Anthocidaris and Echinometra, which was formed within a few minutes after fertilization, looked very hard and maintained the initial size up to the time of hatching (Figs. 18, 19). Although the perivitelline space could be observed until the time immediately before hatching, the space was very small (Figs. 9-11). In the two species, the blastocoel could not be detected to develop before hatching, and the volume of the embryo did not increase in either species until hatching (Figs. $18,19)$.

The formation of blastocoel took place immediately after hatching, as did the embryonic volume increase (Figs. 18, 19). With the enlargement of blastocoel, the embryo became a coeloblastula. The rate of the volume increase was larger in Anthocidaris than in Echinometra (Figs. 18, 19).

The continuous TYPE: This type of the blastula formation is intermediate between the above mentioned types and includes Toxopneustes, Tripneustes, and Pseudoboletia. The blastocoel formation of these three species probably took place at the first cleavage, but the blastocoel was at first detected at the 4th cleavage, partly because of semi-transparency of blastomeres. Concomitant with a slight enlargement of blastocoel, the volume of the embryo increased during cleavage cycles. The rate of the volume increase was, however, higher during the developmental course after hatching than before hatching (Figs. 15-17). The fertilization membrane of the three species reached the full size within a few minutes after fertilization and maintained its size until the time of hatching, similar to the case of the post-hatching type (Figs. 15-17).

In Toxopneustes a rapid increase of the volumes occurred from the 9th cleavage on (Fig. 15). In Tripneustes the volume of the embryo increased rather slightly before hatching (Fig. 16). The perivitelline space became smaller and smaller during cleavage and finally disappeared at the time of hatching (Figs. 5-7). The first phase of hatching in this species was the bulb-out of the embryo through a certain point of fertilization membrane (Fig. 7). The tearing of fertilization membrane by the embryonic wall proceeded, and the embryo showed a successive deformation when hatching out. In Pseudoboletia a small blastocoel was observed at early cleavage stage, but it disappeared at the time of the 8th cleavage. It appeared again after hatching. 
Analysis of the result: The difference between embryo and blastocoel volumes provides an estimate of blastomere volume. As shown in Figures 13-19, the value of the difference in all the seven species studied remained almost unchanged, while the embryonic volume increased. This seems to mean that the total volume of blastomeres is almost constant from the time of fertilization until at least initiation of gastrulation, and that the embryonic volume increase is exclusively due to the increase of the blastocoel. Similarly, the enlargement of the region enclosed by the fertilization membrane in the two species of the pre-hatching type, may be due to the enlargement of embryo.

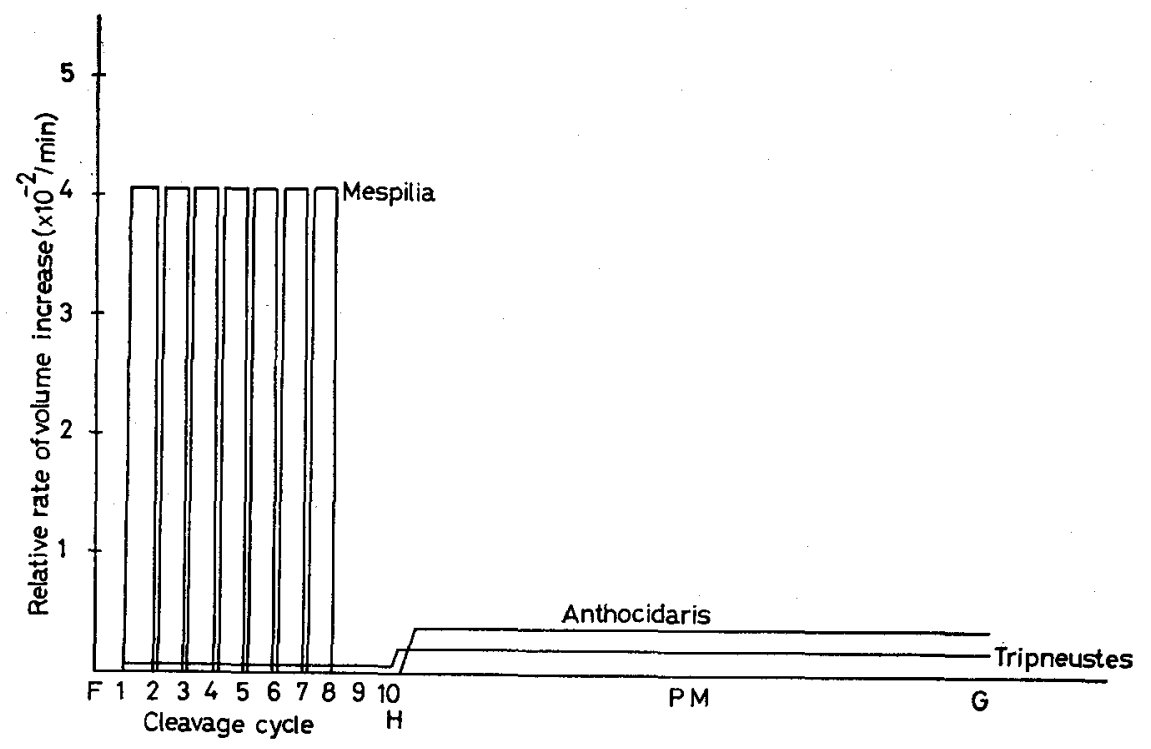

Fig. 20. The relative rate of volume increase during early development of three representative species. Abscissa; developmental stages shown as the time of fertilization (F), cleavage cycles, hatching $(\mathrm{H})$, the appearance of the primary mesenchyme cells (PM) and initiation of gastrulation (G). Ordinate; the relative rate of volume increase $\left(1 / V_{0} \times d V / d t\right)$. The three different pattern of volume increase is clear. The elnargement of embryo in Mespilia occurs in step-wise fashion at each cleavage cycle and the rate of enlargement is considerably higher than the two other species.

In order to compare the three different temporal patterns of blastula formation, the relative rate of embryonic volume increase was estimated in representative three species by

$$
1 / \mathrm{V}_{0} \times \mathrm{dV} / \mathrm{dt}
$$

where $V_{0}$ is the egg volume at the time of fertilization and $d V / d t$ is the rate of embryonic volume increase. The result is shown in Figure 20. The difference in temporal pattern of the volume increase among the three types is clear. The relative rate of the volume increase of Mespilia is approximately ten times higher than that of the two other species. 


\section{Discussion}

The comparative embryological study presented here made clear the three different patterns of blastula formation in sea urchins. One factor that causes such different patterns may be the stiffness both of fertilization membrane and hyaline layer. In the species of the pre-hatching type the stiffness of them may be rather lower, which seems to ensure a rapid and large increase of the volume during cleavage. The enlargement of the blastocoel in these species may be due more to the osmotic pressure developed within the hyaline layer and later within the blastocoel (Dan, 1960) rather than to the change of mechanical packing of blastomeres attached to the hyaline layer (Wolpert and Gustafson, 1961). The stiffness both of fertilization membrane and hyaline layer of the post-hatching type, on the other hand, may be considerably high so that the embryonic volume increase was hardly observed during cleavage. Release from fertilization membrane by hatching, a reduction in the stiffness of hyaline layer by the action of hatching enzyme and the autonomous morphogenetic activity of cells may account for a high rate of the volume increase at the post-hatching blastula stage. This style of enlargement of blastocoel may be mainly due to the change of mechanical and geometrical packing of blastomeres attached to the hyaline layer (Wolpert and Gustafson, 1961). The stiffness both of fertilization membrane and hyaline layer of the continuous type may be intermediate between the above mentioned types.

The present grouping of sea urchins according to the temporal pattern of volume increase coincides with the taxon at the family level. The pre-hatching type is for the family Temnopleuridae, the continuous type for Toxopneustidae, and the post-hatching type for the family Echinometridae. Since the two formers of them belong to the order Temnopleuroida and the latter to the order Echinidea (Yatsu and Uchida, 1972), the taxonomical close relationship exists between the pre-hatching type and the continuous type. As clearly shown in Figure 20, the relative rate of the volume increase of the pre-hatching type is considerably higher than the two other types, while the difference in the relative rate between the post-hatching type and the continuous type is rather small. In addition, the cleavage tempo of the pre-hatching type is higher than the two other types. The tempo is almost the same in the continuous type and the post-hatching type (Maruyama, unpublished data). These facts seem to imply an intimate relation between the species of the continuous type and the post-hatching type at the level of the comparative embryological study, contrary to the taxonomical relationship.

\section{Summary}

1. The patterns of the blastula formation were compared among seven species of sea urchins.

2. According to a temporal ordering, different patterns of the blastula formation were classified into three types, which turn out to be in accordance with the taxa 
at the family level.

a) The pre-hatching type (Mespilia globulus and Temnopleurus reevesi): a rapid and large increase both of embryo and blastocoel volume occurred from the first cleavage until the end of the 7 th cleavage cycle, and thereafter the volume increased very slightly.

b) The post-hatching type (Anthocidaris crassispina and Echinometra mathaei): the blastocoel formation could be detected only after hatching, and the volume increase both of embryo and blastocoel took place after hatching.

c) The continuous type (Toxopneustes pileolus, Tripneustes gratilla and Pseudoboletia maculata): the blastocoel was formed during cleavage and a slight increase both of embryo and blastocoel occurred before hatching. But the main enlargement of embryo took place after hatching.

3. The volume increase of embryo at this stage is exclusively due to that of blastocoel, the total volume of blastomeres remaining rather constant.

Acknowledgement: We wish to express our cordial thanks to Professor Emeritus Katsuma Dan, Tokyo Metropolitan University, for his interest in the present study and reading of the manuscript for publication. Our gratitude is also due to Professor Takashi Tokioka, Associate Professor Eiji Harada and all other staff members of the Seto Marine Biological Laboratory for offering an opportunity to utilize their facilities and giving many useful discussions on the way of this investigation.

\section{REFERENCES}

Dan, K., 1952. Cyto-embryological studies of sea urchins. II. Blastula stage. Biol. Bull., 102: 74-89.

Dan, K., 1960. Cyto-embryology of Echinoderms and Amphibians. Internat. Rev. Cytology, 9: 321367.

Maruyama, Y.K., 1977. Manuscript in preparation.

Monné, L. and S. Hårde, 1951. On the formation of the blastocoele and similar embryonic cavities. Arch. Zool., 1: 463-469.

Wolpert, L. and T. Gustafson, 1961. Studies on the cellular basis of morphogenesis of the sea urchin embryo. The formation of the blastula. Exp. Cell Res., 25: 374-382.

Yatsu, N. and T. Uchida, 1972. "Table of Animal Classification", pp. 680-688. Nakayama-Shoten, Tokyo, in Japanese. 